choice for longitudinal studies in defining the natural history of uremic cardiomyopathy after renal transplantation.

Values are expressed as mean $\pm \mathrm{SD}$ or median (interquartile range). $\mathrm{P}$ Value $<0.05$ demonstrates significance in change of variable following transplantation.

\section{CPEX TESTING DETECTS SUBCLINICAL CARDIAC LIMITATION TO EXERCISE IN EARLY STAGE CKD}

Manvir Kaur Hayer*, Anna Price, Shanat Baig, Boyang Liu, Charles Ferro, Jonathan Townend, Nicola Edwards, Richard P Steeds. Queen Elizabeth Hospital Birmingham

\subsection{6/heartjnl-2017-311726.11}

Introduction Effort tolerance is impaired in end stage kidney disease. Peak oxygen uptake $\left(\mathrm{VO}_{2}\right.$ peak $)$ has been shown to be a powerful predictor of survival in haemodialysis patients. A low $\mathrm{VO}_{2}$ peak and percent predicted $\mathrm{VO}_{2}$ at the anaerobic threshold $\left(\mathrm{VO}_{2} \mathrm{AT}\right)$ have also been associated with excess mortality in patients undergoing kidney transplantation. Data on effort tolerance and cardiovascular disease in early chronic kidney disease (CKD) are very sparse though it is well recognised that cardiovascular mortality begins to increase at a glomerular filtration rate (eGFR) of about $75 \mathrm{ml} / \mathrm{min} / \mathrm{m}^{2}$.

Methods This study examined effort tolerance, cardiac structure and function in 60 patients with CKD (stages 2 to 5) without known cardiovascular disease or diabetes. All patients underwent a cardiopulmonary exercise bicycle test using an individualised ramp protocol. Myocardial ischaemia was excluded by exercise stress echocardiography or $99 \mathrm{~m}$ technetium tetrofosmin single photon electron computed tomography. Lung disease was excluded by formal lung function testing. Cardiac magnetic resonance imaging without gadolinium contrast was used to assess cardiac function and structure. The Kruskall Wallis test was used to compare the difference in mean values across stages of CKD. Correlation coefficients were measured to look for trends between continuous variables.

Results Table 1 shows the baseline characteristics per CKD stage. Percent predicted peak $\mathrm{VO}_{2}$ was negatively associated with eGFR $(\mathrm{r}=-0.358, \mathrm{p}=0.007)$ even after correction for age and haemoglobin $(\mathrm{p}=0.005)$. NT pro-BNP was negatively associated with eGFR $(\mathrm{r}=-0.586, \mathrm{p}=0.001)$, even after similar correction $(\mathrm{p}<0.001)$. The percent predicted $\mathrm{VO}_{2}$ at the anaerobic threshold was also negatively associated with worsening eGFR ( $r=0.282, p=0.039)$. Exercise capacity $\left(\mathrm{VO}_{2} \mathrm{AT}\right)$ was negatively associated with increasing LV mass $(\mathrm{r}=-0.382$, $\mathrm{p}=0.006$ ) but there was no significant association with left ventricular (LV) size, ejection fraction or global longitudinal strain.

Discussion and implications Effort tolerance falls from the earliest stages of CKD in association with a progressive increase in LV mass and NT pro-BNP. This is the first study to examine exercise capacity in patients with early stage CKD in whom coronary artery disease has been excluded, and further study is needed to confirm whether the reduction in exercise capacity is a reflection of diastolic impairment and myocardial fibrosis that characterise end-stage kidney disease.
Abstract 11 Table 1 Baseline demographics across each stage of CKD

\begin{tabular}{llllll}
\hline & CKD & CKD & CKD & CKD & P \\
& $\begin{array}{l}\text { stage 2 } \\
\mathrm{n}=14\end{array}$ & $\begin{array}{l}\text { Stage 3 } \\
\mathrm{n}=17\end{array}$ & $\begin{array}{l}\text { Stage 4 } \\
\mathrm{n}=\mathbf{9}\end{array}$ & $\begin{array}{l}\text { Stage 5 } \\
\mathrm{n}=21\end{array}$ & Value \\
\hline Age (years) & $59(45-67)$ & $65(54-67)$ & $57(53-69)$ & $46(33-58)$ & 0.14 \\
Male & $8(57 \%)$ & $10(59 \%)$ & $6(67 \%)$ & $11(55 \%)$ & 0.912 \\
$\mathrm{Hg}$ (g/L) & $134 \pm 38$ & $135 \pm 11$ & $130 \pm 15$ & $107 \pm 47$ & 0.003 \\
$\mathrm{NT}-$ pro BNP & $68 \pm 70$ & $156 \pm 152$ & $189 \pm 115$ & $631 \pm 651$ & 0.001 \\
EF (\%) & $71 \pm 7$ & $70 \pm 6$ & $68 \pm 10$ & $69 \pm 9$ & 0.913 \\
LVMi (g/m2) & $62 \pm 13$ & $60 \pm 15$ & $62 \pm 9$ & $84 \pm 38$ & 0.01 \\
$\%$ Predicted VO 2 & $84(77-109)$ & $86(82-94)$ & $80(73-88)$ & $73(62-91)$ & 0.019 \\
at Peak & & & & & \\
$\%$ Predicted V0 & $55(42-69)$ & $61(55-66)$ & $49(41-56)$ & $46(39-60)$ & 0.023 \\
at AT & & & & & \\
RER & $1.19 \pm 0.05$ & $1.17 \pm 0.11$ & $1.18 \pm 0.08$ & $1.18 \pm 0.12$ & 0.915 \\
Resting global & $-18.8 \pm 2.1$ & $-18.0 \pm 2.4$ & $-17.9 \pm 1.9$ & $-19.5 \pm 3.1$ & 0.320 \\
longitudinal strain & & & & & \\
(\%) & & & & & \\
\hline
\end{tabular}

Data are presented as median an interquartile range, or mean and standard deviation. A p value of $<0.05$ demonstrates significance at the $95 \%$ confidence interval using the Kruskal Wallis test.

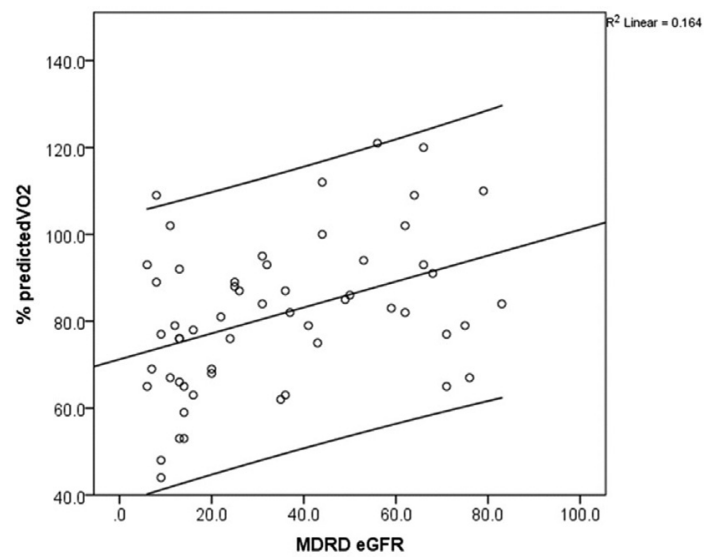

Abstract 11 Graph 1 The association between eGFR and\% predicted $\mathrm{VO}_{2}$. Lines represent the line of best fit and $95 \%$ confidence intervals.

\section{CHARACTERISATION OF CLOZAPINE REFERRALS TO A TERTIARY CARDIOLOGY UNIT}

${ }^{1}$ George Joy*, ${ }^{2}$ Mark Bolstridge, ${ }^{2}$ Eromona Whiskey, ${ }^{3} \mathrm{TA}$ McDonagh, ${ }^{4}$ Sukhi Shergill, ${ }^{5}$ Carla Plymen. ${ }^{1}$ King's College Hospital; ${ }^{2}$ South London and Maudlsey NHS Foundation Trust; ${ }^{3}$ Cardiology Department, King's College Healthcare NHS Foundation Trust; ${ }^{4}$ Institute of Psychiatry, Psychology and Neuroscience, King's College London and Maudsley Hospital London; Imperial College Healthcare NHS Trust

\subsection{6/heartjnl-2017-311726.12}

Introduction Clozapine is an essential tool in the psychiatric armoury: It is the most effective antipsychotic drug and is recommended standard treatment in those with refractory psychotic disorders. The potential risk of cardiac toxicity means 